Sociologie et sociétés

\title{
Quand le trouble amoureux contrarie le masculin
}

La gestion des émotions amoureuses au sein de la Communauté

de la séduction en France

\section{When Love-related Issues Undermine Masculinity Managing Emotions of Love within the Community of Seduction in France}

\section{Mélanie Gourarier}

Volume 46, numéro 1, printemps 2014

Formes d'intimité et couples amoureux

Forms of Intimacy and Couples in Love

URI : https://id.erudit.org/iderudit/1024677ar

DOI : https://doi.org/10.7202/1024677ar

Aller au sommaire du numéro

Éditeur(s)

Les Presses de l’Université de Montréal

ISSN

0038-030X (imprimé)

1492-1375 (numérique)

Découvrir la revue

Citer cet article

Gourarier, M. (2014). Quand le trouble amoureux contrarie le masculin : la gestion des émotions amoureuses au sein de la Communauté de la séduction en France. Sociologie et sociétés, 46(1), 37-57. https://doi.org/10.7202/1024677ar

\section{Résumé de l'article}

Le One-itis est un jeu de mots issu de l'argot américain qui établit une analogie entre les sentiments amoureux et la maladie, la terminologie des pathologies infectieuses se terminant souvent par le suffixe itis. L'usage de cette expression, synonyme d'" obsession amoureuse " s'inscrit dans un contexte spécifique puisque son invention est le fait de jeunes hommes (âgés en moyenne d'une vingtaine d'années) qui se réunissent autour de l'apprentissage de la séduction des femmes. La "Communauté de la séduction ", ainsi que la nomment ceux qui s'y agrègent, est apparue à la fin des années 1990 en Californie dans la mouvance des techniques émergentes du développement personnel et du coaching appliquées à la séduction masculine. Fondée sur l'examen empirique des pratiques et des discours amoureux formulés par les jeunes hommes rencontrés durant l'enquête, cette contribution entend penser les modalités de l'articulation entre l'expérience sentimentale douloureuse, la normalisation et la hiérarchisation des masculinités.
Tous droits réservés ㄷ Les Presses de l’Université de Montréal, 2014
Ce document est protégé par la loi sur le droit d'auteur. L'utilisation des services d'Érudit (y compris la reproduction) est assujettie à sa politique d'utilisation que vous pouvez consulter en ligne.

https://apropos.erudit.org/fr/usagers/politique-dutilisation/ 


\section{Quand le trouble amoureux contrarie le masculin}

La gestion des émotions amoureuses au sein de la Communauté de la séduction en France

\section{MÉLANIE GOURARIER}

Cresppa

Centre de recherches sociologiques et politiques

de Paris

59-61, rue Pouchet

75849 Paris Cedex 17

France

Courriel: melanie.gourarier@ehess.fr

C I la "MAladie D’Amour» est un thème littéraire et social récurrent bien antérieur $\checkmark$ à l'époque romantique (Lafayette, 2009 [1678]; Goethe, 1999 [1774]), au point que l'on peut se demander si l'on n'a jamais parlé de l'amour sans le superposer à l'idée de souffrance, l'expérience de cette douleur et le sens qui lui est accordé sont nécessairement variables et socialement situés. Le désarroi amoureux, épisode aussi désagréable qu'ordinaire, doit ainsi faire l'objet d'un examen critique, ce qui passe en priorité par son historicisation. Depuis l'exhortation de Lucien Febvre à s'intéresser à la "sensibilité dans l'histoire» (Febvre, 1943), d'importants travaux ont contribué à façonner une histoire des émotions (Elias, 1973; Delumeau, 1978), dont certains se sont plus directement centrés sur l'expression des sentiments amoureux (Duby, 1988; Bologne, 2007; Rougemont, 1972). Rares sont toutefois les auteurs qui ont adopté la perspective de Ute Frevert suivant laquelle «les émotions ne sont pas seulement fabriquées par l'histoire, elles fabriquent aussi l'histoire» (Frevert, 2009: 202)1 ${ }^{1}$. Si les émotions participent d'un processus historique, ne faut-il pas penser également les sentiments comme des «acteurs majeurs de l'histoire» (Illouz, 2006: 17) ? C'est là l'ambition de sociologues

\footnotetext{
1. Traduit de l'allemand par Eva Illouz (Illouz, 2012: 25).
} 
telles Arlie Hochschild dont les recherches portent sur le «travail émotionnel» (1983; 2003) ou d'Eva Illouz sur les «sentiments du capitalisme» (2006). Dans la perspective d'une sociologie des émotions, cet article vise à saisir la manière dont les sentiments amoureux ne sont pas seulement produits par le social mais transforment les subjectivations, en l'occurrence ici l'expérience et les devenirs masculins.

Surreprésentée dans les ouvrages de psychologie populaire ${ }^{2}$ et dans les médias, notamment dans la presse féminine à travers la question du célibat posée comme un "problème» social contemporain, la souffrance amoureuse reste peu travaillée par les sciences sociales. D'importants ouvrages sont pourtant parus ces dernières années autour de Jean-Claude Kauffman sur le célibat (1999), de François de Singly sur la séparation (2011) ou, plus récemment, d'Eva Illouz qui aborde plus directement les affects douloureux qui résultent de l'expérience amoureuse (2012). Cependant, ces derniers se concentrent sur ce que vivent les femmes au risque de réifier une vision de l'amour conçue comme une "question féminine». Or, ce que montre cet article, c'est que les hommes — du moins ceux de la génération sur laquelle repose l'enquête parlent volontiers de leurs émotions amoureuses, d'autant plus qu'ils les conceptualisent. Cet article entend prendre au sérieux les déboires amoureux des hommes et s'intéresse plus particulièrement à ce qu'ils en disent. Quels sens accordent-ils à leurs expériences amoureuses malheureuses? Comment lient-ils cette expérience à l'épreuve de la masculinité? Et, sur quels modes l'expriment-ils à l'enquêtrice ${ }^{3}$ ?

\section{LA COMMUNAUTÉ DE LA SÉDUCTION}

Pour répondre à ces questions nous nous appuierons sur les résultats d'une enquête ethnographie conduite en France entre 2007 et 2010 (Gourarier, 2012) auprès d'un groupe composé exclusivement d'hommes qui ambitionnent d'apprendre à séduire les femmes en adaptant les méthodes du coaching et du développement personnel à ce nouvel effet. Apparue à la fin des années 1990 en Californie avant de se développer dans toute l'Amérique du Nord puis en Europe.

La Communauté de la séduction, originairement Seduction Community, ainsi que la nomment les individus qui s'y agrègent, a bénéficié de l'essor concomitant des réseaux sociaux sur Internet, du développement personnel et plus spécialement $\mathrm{du}$ coaching. À ses débuts, la Communauté de la séduction était un groupe de parole entre hommes organisé autour de la figure emblématique de Ross Jeffries - qui, pour l'anecdote, a inspiré le personnage du coach joué par l'acteur américain Tom Cruise dans le film Magnolia (Anderson, 2000) — formé aux techniques à la fois de la programmation neurolinguistique (méthode de psychothérapie pragmatique) et de l'hypnose. Voués initialement à l'expression du malaise masculin et aux difficultés

2. À titre d'exemple, on citera ici le best-seller de John Gray, Les hommes viennent de Mars, les femmes viennent de Vénus (1997 [1992]).

3. Sur les effets de la relation d'enquête entre une ethnologue et des enquêtés, jeunes hommes apprentis séducteurs hétérosexuels, voir Gourarier (2007, 2011, 2012). 
rencontrées par les hommes dans leur expérience de l'hétérosexualité, les groupes de parole se sont rapidement transformés en séminaires et ateliers payants ${ }^{4}$, animés par ceux qui se présentaient comme les plus expérimentés et les plus érudits, chargés de transmettre leurs connaissances en matière de séduction aux débutants qui occupent le statut d'initiés. Les compétences à acquérir en matière de séduction correspondent alors à des compétences évaluées comme spécifiquement masculines telles que la détermination, la force (morale et physique), le pouvoir ou la mesure (Gourarier, 2012). Pour y parvenir, les apprentis séducteurs suivent un apprentissage de la séduction des femmes à la fois théorique, par le biais de séminaires qui prennent la forme de cours magistraux, et pratique, en allant sur le terrain pour s'entraîner, en petit groupe, aux techniques de séduction «en situation » dans la rue, les bars, les transports publics ou les centres commerciaux auprès de femmes choisies au hasard qui ignorent tout du dispositif.

À ce premier développement du groupe autour du coaching se juxtapose une pratique axée sur les sociabilités entre hommes à partir des nouvelles technologies de l'information et de la communication, et plus particulièrement des réseaux sociaux sur Internet. En 1994, un groupe d'élèves de Ross Jeffries, férus d'informatique, décide d'ouvrir la première liste de diffusion sur Internet de la Communauté de la séduction, alt.seduction.fast, dans le but de favoriser la circulation des informations entre les individus qui participaient alors aux différentes rencontres. Ces groupes de discussion ont surtout permis l'extension de la Communauté à l'échelle internationale par le biais de l'ouverture de sites et de forums Internet favorisant l'échange entre les membres sur le plan local afin de prolonger hors ligne les sociabilités nouées en ligne. De cette manière est apparu en 2004 le premier site Internet français du groupe revendiquant, dans l'appellation même, une spécificité culturelle: Frenchtouchseduction.com. Comparativement aux méthodes de séduction américaines évaluées comme reprenant de façon trop littérale et schématique les techniques du développement personnel issues du management, les membres français du groupe prescrivent une manière «toute française» de séduire les femmes, inspirée d'une certaine tradition romanesque de l'«art de séduire» (Monneyron, 1997), fondé sur le «charme» du séducteur, son éloquence, sa culture et sa capacité d'improvisation.

La Communauté de la séduction s'est ainsi structurée autour de deux pratiques distinctes. D’une part, les activités du groupe sont axées sur les échanges entre hommes dans le but de les faciliter et de les densifier (réunions régulièrement organisées sous la forme de séminaires, systèmes d'échanges par messagerie numérique sur les sites Internet dédiés, etc.). D’autre part, le système d'apprentissage de la séduction mis en place par le biais du coaching correspond à un processus de masculinisation. De sorte que cette organisation, bien qu'a priori vouée à la séduction des femmes, est, comme a permis de le préciser l'enquête, consacrée aux sociabilités masculines et au devenir

4. Les séminaires, coaching et relooking coûtent à ceux qui y souscrivent de 25 à plus de 5000 euros en fonction de la durée et de la renommée nationale ou internationale du coach qui prodigue ses conseils (Gourarier, 2012:363). 
masculin. L'apprentissage de la séduction des femmes auquel les membres de ce groupe se consacrent devient ainsi moins un enjeu qu'un prétexte pour se retrouver entre hommes.

\section{L'ENQUÊTE}

L'enquête a porté sur un groupe de jeunes hommes âgés de 18 à 35 ans vivant en Îlede-France ainsi qu'en région (principalement à Nice, Nantes, Lille et Marseille). A minima, les individus rencontrés durant l'enquête sont bacheliers, vivent au domicile parental, conjugal, seuls ou en colocation. Ils proviennent pour la plupart des classes moyennes. Ils sont étudiants ou exercent des professions aussi diverses que l'enseignement, le service, l'ingénierie, le management, le conseil, l'informatique ou la gestion. Si la formation à la séduction par le biais du coaching peut entraîner un coût élevé dans la mesure où il peut atteindre entre 200 et 5000 euros en fonction de la renommée du coach qui prodigue ses conseils et de la durée de l'enseignement, les membres qui n'ont pas la possibilité de dépenser de telles sommes ne sont toutefois pas exclus de cet apprentissage dans la mesure où la plupart des articles, ouvrages ainsi que certains cours sont mis à disposition librement sur Internet ou s'échangent entre membres. De fait, la composante sociale du groupe est relativement hétérogène.

L'ethnographie repose sur une immersion longue au sein des espaces où les enquêtés se réunissent à Paris (cafés, salles de réunion, palais des congrès, espaces publics et domicile de certains des enquêtés), sur une série d'entretiens semi-directifs répétés de type biographique et limités à une dizaine d'interlocuteurs, sur une observation des forums Internet de discussion ${ }^{5}$ ainsi que sur un archivage des articles, blogues et autres documents numériques ou manuscrits produits par les enquêtés. Cet article mobilise d'une part des extraits d'entretiens recueillis en face à face avec un petit groupe d'enquêtés rencontrés sur le terrain et, d'autre part, des messages de forums Internet postés par les membres de la Communauté de la séduction. Si les individus prennent un pseudonyme au sein de la Communauté, pour des questions d'anonymisation, nous avons choisi de les modifier dans les extraits restitués.

Au regard du contexte étudié, on pourrait s'étonner de voir consacrer un article au malaise amoureux masculin résultant de relations avec les femmes, d'après l'étude d'un groupe qui semble privilégier les rapports entre hommes ainsi que l'enquête le laisse apparaître. Or, ce sont précisément ces circonstances spécifiques qui permettent de poser la problématique. Dans un contexte où les liens entre hommes priment sur les relations hétérosexuelles, qu'en est-il des émotions amoureuses à l'endroit des femmes? Au sein de la Communauté de la séduction, ces émotions font l'objet d'une attention particulière, appréciable dans les discours prolifiques que nous nous proposons d'analyser.

5. Les principaux sites Internet consultés durant l'enquête sont: Frencheseduction.com, Spikeseduction.com, Verselejus.com 


\section{L'expérience ethnographique}

L'enquête ethnographique conduite au sein de la Communauté de la séduction en France repose sur une pluralité des modes de recueil des données pensés non plus comme des séquences distinctes, mais continues et imbriquées. C'est pourquoi, dans ce travail, l'expression «d'observation participante» est remplacée par celle d' "expérience ethnographique», plus apte à saisir les effets consécutifs des techniques adoptées. L'expérience étant la «connaissance des êtres et des choses par leur pratique et par une confrontation plus ou moins longue de soi avec le monde ${ }^{6} »$, il s'agit de penser l'ethnographie au-delà des méthodes successivement convoquées pour la définir. Ainsi, les mois passés sur le terrain à participer aux séminaires et séances de coaching en séduction ont favorisé l'instauration de relations propices à la mise en place d'entretiens approfondis et non directifs avec un petit nombre d'apprentis séducteurs (8) et de coachs (4), tandis que la tenue de ces entretiens avait simultanément une influence sur la socialisation de l'enquêtrice au sein du groupe $^{7}$. D'une part, dans la mesure où les entretiens se sont répétés sur plusieurs mois, parfois plusieurs années, les liens noués avec les interviewés prenaient peu à peu la forme de l'amitié. D'autre part, les liaisons privilégiées entretenues avec certains valaient méfiance et inimitié des uns en même temps que la sympathie des autres. Ainsi l'entretien n'était pas un espace clos et préservé du terrain mais influait sur les modalités de son maintien. Le recueil de la littérature grise a procédé également de l'expérience ethnographique dans la mesure où l'apprentissage et le partage des données sur la séduction sont les activités principales du groupe étudié. La circulation des données, à laquelle nous avons participé en partageant des articles avec les membres du groupe ou en discutant sur les forums, a favorisé non seulement notre compréhension des savoirs du groupe, mais aussi l'intégration au terrain en nous insérant dans un réseau d'échanges des connaissances.

Partant des discours amoureux formulés par les jeunes hommes rencontrés durant l'enquête, nous discuterons de la relation entre l'expérience sentimentale qualifiée de douloureuse, la fabrication du masculin et la hiérarchisation des masculinités. Pour parvenir à qualifier le malaise amoureux tel qu'il est exprimé au sein de la Communauté de la séduction et ses effets sur la définition et la distinction des masculinités, il faut d'abord revenir sur le contexte de sa formulation. Les jeunes hommes sur lesquels a porté l'enquête envisagent les relations de séduction et plus globalement les rapports amoureux hétérosexuels actuels comme «abîmés» par une marche vers l'égalité des sexes qui serait à présent « dépassée».

6. Source: «Expérience», www.cnrtl.fr/definition/exp \% C3\% A9rience (page consultée le 19 septembre 2011).

7. La négociation de ma présence en tant que femme sur un terrain d'hommes apprentis séducteurs a fait l'objet d'un précédant article (Gourarier, 2011). 


\section{S'AIMER EN CONTEXTE «POST-ÉGALITAIRE »}

Nous ne devons pas considérer la liberté ou l'égalité comme l'étalon suprême pour mesurer les transformations sociales. Il faut au contraire enquêter précisément sur la façon dont les nouvelles formes d'égalité ou de liberté ont transformé la «texture affective» des relations intimes et peuvent aller à l'encontre l'une de l'autre. (Illouz, 2006: 61)

La réinvention de la séduction à laquelle se consacrent les apprentis séducteurs se situe dans une conjoncture spécifique dans laquelle ils pensent se situer: celle d'une égalité acquise entre les sexes résultant d'un processus historique, sociologique, politique et économique qui serait aujourd'hui parfaitement entériné. Âgés d'une vingtaine d'années pour la grande majorité d'entre eux (Gourarier, 2012: 136), les membres de la Communauté de la séduction en France se définissent comme les "enfants des révolutions sexuelle et féministe ${ }^{8}$ ». Dans ce contexte, l'égalité des sexes est pour eux la condition sociale «normale» et constitue une norme "parfaitement» incorporée ${ }^{9}$ : ils n'auraient connu qu'elle. S'ils évoquent la question des inégalités entre les hommes et les femmes et discutent de la domination masculine, c'est pour les conjuguer uniquement au passé, comme des paradigmes lointains, correspondant à un temps historique révolu. La détermination de ce contexte égalitaire est un processus qui a été identifié, notamment par Christine Delphy, sous le concept du «mythe de l'égalité déjà là» (Delphy, 2010). Or, postuler que l'égalité serait accomplie a au moins deux effets. D'une part, sous l'affirmation d'une égalité acquise, les inégalités effectives s'effacent. D'autre part et consécutivement, les revendications féministes apparaissent dès lors dépassées et s'en trouvent très largement dépréciées, notamment dans les discours médiatiques et dans ceux issus des ouvrages de psychologie populaire ( $p o p$ psychology $)^{10}$. Appeler à l'égalité tandis que celle-ci serait accomplie confère en effet aux discours féministes un caractère inique et dangereusement excessif. Si les jeunes hommes interviewés durant l'enquête considèrent que le féminisme a pu être un bien par le passé, ayant contribué à l'amélioration non seulement de la condition féminine, mais aussi des relations hommes/femmes, notamment grâce à l'indépendance sexuelle et économique acquise à ces dernières - indépendance, envisagée toutefois principalement comme une libération des obligations masculines et une amélioration de l'accessibilité à la sexualité des femmes (Bozon, 2001), cependant tous considéraient la pérennité du féminisme comme particulièrement néfaste dans la mesure où les féministes d'aujourd'hui seraient allées trop loin. Ne se contentant pas de l'égalité, elles travailleraient à la féminisation du champ social et à la domination féminine. Sur le forum de discussion d'un des sites de la Communauté de la séduction, un internaute s'interroge:

8. Cette expression a été employée par un des enquêtés, Oryx, alors âgé de 37 ans, à l'occasion d'une discussion informelle au cours de laquelle il souhaitait expliquer la singularité du contexte dans lequel il avait grandi.

9. Dans sa thèse, qu'elle consacre à l'étude de la séduction en Norvège et en France, Fanny ForgeauZerbib réserve une partie à la question de l'égalité incorporée (Forgeau-Zerbib, 2001).

10. Sur cette question, voir notamment Illouz (2006) et Jonas (2006). 
«Serions-nous dans une société misandre?» (Spikeseduction.com, consulté le 15 juin $2013^{11}$ ). D'après lui, le féminisme aurait conduit à « une société d'hommes frustrés et dominés par les femmes, il ne serait donc plus question d'un échange d'égal à égal, puisqu'on filerait à toute allure vers une société de bourreaux psychologiques en talons aiguilles» (ibid.). Dans cette société gouvernée par les femmes et le féminin, les hommes n'auraient plus leur place et seraient affaiblis par le processus de féminisation comme l'avance un second internaute:

La féminisation de la société me fait gerber. Les hommes sont devenus des pleureuses, apeurées par les femmes, ou des larves incapables de gagner le respect du sexe opposé (et tout le monde sait qu'une femme ne peut aimer son mari sans l'admirer). Je fais absolument mienne cette citation de Nietzsche: «L'homme doit être élevé pour la guerre et la femme pour le délassement du guerrier: tout le reste est folie.» (Spikeseduction.com, page consultée le 23 juillet 2013)

Les membres de la Communauté de la séduction pensent ainsi la «condition masculine» actuelle dans un contexte spécifique où le maintien des revendications féministes participerait à inverser le sens du pouvoir entre les sexes. Le paradigme qu'ils définissent n'est donc plus égalitaire, mais post-égalitaire. On assisterait donc à une révolution inégalitaire opérant un renversement du sens de la domination puisque les oppresseurs d'hier seraient les opprimés d'aujourd'hui. Cette inversion paradigmatique procède d'un discours masculiniste, fondé sur la détermination du groupe des hommes comme groupe minorisé, menacé, dont les intérêts sont, dès lors, à défendre. Nous proposons ainsi de qualifier de masculinisme toute pensée axée sur la subjectivité masculine, concédant aux hommes la place de victime - et de masculiniste, toute personne ou groupe de personnes travaillant à défendre la «cause des hommes » et à améliorer la «condition masculine» dans un mouvement de confrontation réactive vis-à-vis des femmes en général et du féminisme en particulier. Le masculinisme a ainsi pour particularité non seulement de réifier le féminisme en s'opposant à lui, mais surtout de constituer les hommes en groupe social conscientisé, résistant aux femmes et développant des revendications proprement masculines, conservatrices de leurs prérogatives. Or, pour les membres du groupe étudié ici, l'un des moyens les plus efficaces de la défense des prérogatives masculines est la renégociation des positions hommes/femmes dans les rapports amoureux et de séduction.

Pour retrouver la position hégémonique qu'ils prétendent avoir perdue, les apprentis séducteurs entendent se désengager des relations avec les femmes dans la mesure où ces dernières tiendraient les hommes par les émotions sexuelles et amoureuses qu'elles suscitent, ainsi que l'explique un des interviewés.

11. Afin de préserver l'anonymat des auteurs des articles et messages publiés sur les forums Internet de la Communauté de la séduction, seul le site de référence est mentionné. Par ailleurs, certains extraits reproduits dans cet article ont été publiés dans des espaces Internet privatifs, dont l'accès est limité par les modérateurs. 
La séduction des femmes est super puissante [...]. C’est bien connu qu'avec ça elles font tourner le monde. Pour moi, c'est vraiment l'origine du problème. Le problème, c'est surtout que maintenant elles ont tout gagné et qu'elles ont tous les pouvoirs. (Sam, 19 ans, étudiant en arts appliqués, région parisienne)

La séduction qu'exercent les femmes sur les hommes serait donc ici à l'origine du "problème». En apprenant à se désaffecter des charmes et des amours féminins, les jeunes hommes pensent ainsi rétablir un ordre du genre qui se serait dissous avec les révolutions féministes. L'amour envers les femmes est dès lors chargé d'une valeur symbolique négative qu'ils opposent à l'amitié entre hommes, affection largement promue par les jeunes hommes interviewés.

\section{DES AFFECTIONS ASYMÉTRIQUES}

Au sein de la Communauté de la séduction, l'amour hétérosexuel est explicitement relégué au second plan. La valeur que lui concèdent les jeunes hommes rencontrés durant l'enquête est infériorisée comparativement à la valeur que revêtent les amitiés entre hommes. L'analyse des discours révèle en effet que l'amour envers les femmes fait l'objet d'une forte dépréciation visant en miroir la valorisation de l'amitié masculine. C'est ce qu'illustre un commentaire posté sur un forum du principal site français de la Communauté de la séduction à l'occasion d'une discussion consacrée à l'amitié entre hommes:

Les amis, c'est une chose très rare et pour moi la valeur de l'amitié n'a pas de prix! Largement au-dessus de n'importe quelle Hot Babe [femme désignée comme particulièrement désirable ${ }^{12}$ ! (Frenchtouchseduction.com, page consultée le 10 mars 2012)

Ce commentaire est significatif dans la mesure où la question de la valeur des sentiments est évoquée de façon très explicite. L'amitié entre hommes - qui n'a pas de prix - aurait plus de valeur que l'amour pour les femmes. L'incommensurabilité de l'amitié entre hommes s'explique ici par sa rareté, comparativement à l'amour des femmes, y compris pour celles qui sont évaluées comme les plus désirables. La notion de rareté est également apparue lors d'une discussion conduite sur un forum Internet avec l'un des membres du groupe, Oryx, le plus âgé des enquêtés puisqu'il avait 35 ans lorsque nous l'avons par la suite rencontré. Psychoclinicien enseignant dans de grandes écoles militaires, Oryx fait figure d'autorité au sein du groupe. Dans l'extrait qui suit, il compare ses relations d'amitié qu'il estime précieuses aux rapports avec les femmes qu'il juge dérisoires:

12. La Communauté de la séduction emploie un vocabulaire spécifique constitué d'anglicismes, d'abréviations et d'acronymes. Des lexiques sont consultables sur les principaux sites Internet du groupe. Voir par exemple: Frenchtouchseduction.com/lexique. L'expression Hot Babe désigne ainsi: «Une femme sexy. Femme extrêmement désirable, type playmate. Se dit à l'origine d'une personne présentant une notation globale très forte $(8+$ sur 10$)$ [renvoie ici à la classification de 0 à 10 de la désirabilité des femmes désignées par les apprentis séducteurs]. Désigne les cibles les plus convoitées» (Naturaltechnics.com, page consultée le $1^{\mathrm{er}}$ mars 2012). 
Nous étions de vrais amis car nous avions vécu certaines épreuves ensemble. L'amitié virile ne surgit que très rarement des mondanités, car elle demande une certaine admiration réciproque et se nourrit d'actions en commun, pas de bla-bla, qui lui, suffit avec les femmes... C'est ce qui en fait toute la valeur; voilà pourquoi les femmes accordent une grande valeur à celui qui a au moins un véritable ami, en phase de séduction, et c'est aussi pour cela que bien souvent en LTR [Long Term relationship (relation longue)] elles cherchent à nous en éloigner, car elles ne peuvent pas rivaliser avec cette amitié-là.» (Frenchtouchseduction.com, page consultée le 15 juin 2012)

Émotion concurrente et opposée à l'amitié entre hommes, l'amour des femmes freinerait l'épanouissement des liaisons masculines envisagées ici comme moralement supérieures. En effet, pour Oryx, l'amitié masculine est celle de l'effort et du travail des sentiments. "Profonde» et "authentique», elle ne tolère pas les «bla bla», discours vides de sens et d'émotions dont les communautaires usent pour séduire les femmes. Les «bla bla» désignent ici de façon dépréciative à la fois la parole avec les femmes et la parole des femmes. La hiérarchisation des liens entre hommes et entre hommes et femmes trouve par ailleurs son explication dans la différenciation sexuée des émotions. Les émotions féminines, trop aisément attisées et donc superficielles, auraient de fait moins de valeur que celles masculines suscitant les seules «véritables» amitiés. Dans la pensée commune moderne et contemporaine, les femmes seraient en effet plus sentimentales et naturellement enclines aux choses de l'amour (Illouz, 2012). D'après les discours recueillis lors de l'enquête, les femmes tomberaient amoureuses «trop » facilement, ce qui aurait pour effet d'amoindrir la qualité de leurs affections qui se porteraient indifféremment sur n'importe quel objet. Ce sont ainsi les capacités émotionnelles des femmes qui se trouvent dévaluées par les apprentis séducteurs qui les envisagent comme nécessairement triviales comparées à celles des hommes, plus «authentiques» et «vertueuses» par l'excellence des qualités morales qu'ils investissent dans l'amitié masculine. Pour cette raison, ainsi que l'illustre l'extrait d'entretien précédemment cité, les femmes sont susceptibles de jalouser la relation privilégiée entre un homme et son «véritable ami» car non seulement elles en sont exclues, mais les sentiments de type amoureux qu'elles suscitent ne pourront jamais atteindre l'intensité du lien entre hommes. Pour les jeunes hommes interrogés, les femmes auraient dès lors tout intérêt à entraver le développement des amitiés masculines, en détournant l'attention de ces derniers par leur séduction ${ }^{13}$. C'est ce qu'illustre un extrait d'entretien mené avec Lynx — l'un des coachs de la Communauté de la séduction en France - , au cours duquel il oppose la séduction «naturelle» des femmes à l'apprentissage nécessaire de la séduction pour les hommes qu'il conçoit comme un moyen du rééquilibrage du rapport de sexe:

13. La dangerosité de la séduction féminine est un thème classique de la littérature que l'on trouve déjà dans l'Antiquité grecque incarnée par les Sirènes dans l'Odyssée. La séduction de leur chant est fatale aux navigateurs qui en perdent le sens de l'orientation pour venir s'échouer sur les récifs où ils étaient dévorés par ces êtres mythiques mi-femmes, mi-oiseaux (Homère, XII, 39). 
- C'est bien connu que les femmes font tourner le monde... Tu sais, tu retrouves ça dans toute la littérature classique. Avec leur séduction naturelle, elles nous font perdre la tête! Elles font perdre la tête aux hommes et peuvent même les conduire à la ruine!

- À la ruine?

- Oui, moi j'ai pu observer ça chez des potes tellement accros à des nanas qu'ils en oublient tout le reste, leurs potes, leur boulot, leur famille, etc. [...] C'est ce qui me motive, tu vois, dans mon métier de coach, c'est que je permets à ces gars de voir qu'eux aussi ils peuvent avoir ce pouvoir [...] plus naturellement que possèdent les femmes.» (Lynx, 34 ans, coach au sein de la Communauté de la séduction et expert en système d'information, région parisienne)

La «domination» affective et sexuelle qu'exerceraient les femmes sur les hommes est ainsi appréhendée comme un trouble psychique, ces derniers allant jusqu’à "perdre la tête» et négligeant d'autres champs importants de leur vie d'homme: leur carrière, leurs amis, etc. Ainsi requalifié comme un mal, l'attachement amoureux hétérosexuel est pris en charge au sein de la Communauté de la séduction afin de rétablir un état émotionnel constitutif d'un sujet masculin «normal» et accompli grâce aux techniques du développement personnel.

\section{L'AMOUR HÉTÉROSEXUEL COMME TROUBLE MASCULIN : LE ONE-ITIS}

Craint et déprécié en fonction de l'intensité des sentiments masculins engagés, l'amour envers les femmes prend ainsi la forme d'un trouble que les membres de la Communauté de la séduction choisissent d'appeler le One-itis. Le One-itis est un jeu de mots issu de l'argot américain qui vise à établir une analogie entre les sentiments amoureux et la maladie, la terminologie des pathologies infectieuses se terminant souvent par le suffixe itis. Tel est le cas par exemple du terme bronchitis (bronchite). À travers la conceptualisation du One-itis, l'émotion amoureuse se trouve ainsi requalifiée en pathologie psychique. Un article publié sur l'un des principaux sites Internet du groupe propose de définir ainsi le sens du One-itis:

Obsession amoureuse.

[...] Être atteint d'une obsession amoureuse qui vous fait dire «elle est unique, elle est parfaite, il n'y a qu'elle qui vaille le coup sur terre, je ne veux qu'elle», est ainsi décrit comme une véritable maladie [...]. Son obsession et sa frustration [du sujet masculin touché par le One-itis] vont le pousser à la trouver absolument unique et spéciale, et va progressivement se persuader que s'il «rate» cette fille, alors il aura loupé la meilleure femme au monde, avec le désespoir qui s'ensuit. Évidemment, c'est du grand n'importe quoi, mais le One-itis fait perdre toute mesure. L'obsession amoureuse entraîne une totale perte de lucidité vis-à-vis de cette fille. (Frenchtouchseduction, page consultée le 31 juillet 2013)

Ainsi le One-itis est un synonyme de l'obsession amoureuse comme émotion anormalement excessive qui ferait perdre tout sens de la mesure et altérerait la capacité de jugement. C'est en cela que le One-itis passe pour un trouble psychique dans la mesure où il aurait pour effet d'embrouiller la conscience de celui qui en serait touché. L'un 
des principaux objectifs de l'apprentissage de la séduction est alors de permettre aux hommes de se prémunir de ce mal, ce qui passe par la connaissance préalable de ses causes comme l'indique le titre d'un article publié sur l'un des sites Internet du groupe: «Le One-itis, le comprendre pour le maîtriser», dont voici un extrait:

Dans des situations extrêmes, le One-itis peut conduire à une dépression violente, à une dégradation progressive de sa propre personnalité complètement absorbée par l'obsession de l'autre. On peut expliquer le One-itis par les raisons suivantes pour un homme:

- L'homme n'a pas suffisamment confiance en lui et notamment dans sa capacité à séduire les femmes, les quelques rencontres qu'il arrive à faire prennent alors beaucoup d'importance, il place la femme avant lui-même.

- L'homme particulièrement [le débutant] idéalise une femme et la place au- dessus de tout, il pense assurément qu'il trouvera son bonheur dans la relation qu'il espère avec cette femme.

- L'homme a une faible expérience des femmes (voire inexistante) et ne réalise pas qu'il existe des millions de femmes sur terre.

- L'homme est trop sensible, souvent romantique, il souffre facilement émotionnellement.

- L'homme est en manque d'affection, il réclame de l'amour et de l'attention à son égard.

- L'homme a trouvé une femme qui lui correspond parfaitement ou une femme à laquelle il a toujours rêvé (très forte attraction), par manque de confiance, il a peur de perdre ce précieux objet.

- L'homme s'est laissé contrôlé dans la relation au point d'en devenir dépendant, il s'est entièrement sacrifié dans la relation. (Verselejus.com, page consultée le 23 juin 2013)

La description qui est faite ici des facteurs déclencheurs du One-itis permet de distinguer un sujet type, tout particulièrement enclin à développer ce qui est évalué lors de l'enquête comme relevant de l'obsession amoureuse. Le sujet du One-itis est d'abord un homme qui aurait peu d'expérience hétérosexuelle. Ce dernier n'aurait entretenu que de rares relations affectives et sexuelles avec les femmes. La rareté de ces expériences le conduirait à trop considérer les femmes et de ce fait, à s'en intimider, ce qui passe, au sein du groupe étudié, pour un comportement contreproductif en matière de séduction masculine. En effet, il apparaît dans cet article qu'une des prescriptions majeures faites aux apprentis séducteurs est d'accorder plus d'importance «à soimême» qu'à la femme convoitée: il ne faut pas placer «la femme avant [soi-] même» (ibid.). Il s'agit de ne pas «s'oublier» dans une relation naissante ou déjà là, en privilégiant ses désirs et besoins, y compris au détriment de la partenaire. Cette injonction doit être saisie dans la perspective d'une recommandation plus globale faite aux apprentis séducteurs de s'affirmer vis-à-vis des femmes en maintenant le pouvoir dans la relation. La «sensibilité», le «romantisme» ou l' «émotivité» sont des émotions qui passent pour être spécifiquement féminines et contredisent dès lors l'affirmation du sujet masculin en l'exposant à un attachement amoureux hétérosexuel excessif. Dans ces conditions, la trajectoire des séducteurs en devenir vise l'apprentissage de la gestion 
de ce que l'on qualifiera ici, à la suite d'Eva Illouz, de «capital émotionnel» (Illouz, 2006).

\section{GÉRER SON CAPITAL ÉMOTIONNEL}

Dans son ouvrage, Les sentiments du capitalisme, Eva Illouz (2006) emprunte au vocabulaire bourdieusien la notion de capital pour l'appliquer aux champs des émotions. D'après elle, "les champs émotionnels fonctionnent non seulement en créant et en élargissant le domaine du pathologique et en commercialisant la santé émotionnelle, mais aussi en réglant l'accès à de nouvelles formes de compétence sociale que je qualifierai de compétence émotionnelle. De la même façon que les champs culturels sont structurés par la compétence culturelle [...], les champs émotionnels sont structurés par la compétence émotionnelle, la capacité à manifester le style émotionnel défini et promu par les psychologues» (Illouz, 2006: 118) et d'autres acteurs que l'on peut regrouper dans le marché du self-help auquel participe la Communauté de la séduc$\operatorname{tion}^{14}$.

Par l'intermédiaire du coaching, la Communauté de la séduction développe tout un dispositif qui vise l'apprentissage de la gestion des émotions, gestion perçue comme nécessaire au devenir masculin. De la sorte, si le One-itis est un trouble qui frappe prioritairement les hommes, il ne touche pas tous les hommes de la même manière et concerne une certaine catégorie d'hommes, le débutant, celui qui commence son apprentissage. Durant l'enquête, le One-itis est en effet apparu comme un élément déclencheur de l'adhésion au groupe. Pensant souffrir d'une obsession amoureuse, les jeunes hommes interviewés expliquent leur entrée dans la Communauté de la séduction par le fait qu'ils cherchaient alors un remède à ce qu'ils qualifient comme un «trouble». Un extrait d'entretien conduit avec Sam illustre bien comment ce dernier associe son entrée et sa trajectoire au sein de la Communauté de la séduction à un processus d'affranchissement vis-à-vis d'une certaine «dépendance» amoureuse:

- Peux-tu me décrire précisément ton premier contact avec la Communauté de la séduction, comme tu t'en souviens?

- Le premier contact? Toute une histoire pour tout le monde. Pour ma part, ça s'est fait de manière assez prosaïque. Je m'étais pris une grosse latte d'une fille que j'avais harcelée, la pauvre, et j'ai tapé «comment séduire une fille» sur Google. Je suis tombé sur FTS [Frenchtouchseduction.com] et je n'en suis jamais vraiment ressorti.

- Tu veux dire que c'est pour séduire cette fille que tu t'es d'abord intéressé à la séduction?

- J'étais obsédé par cette fille, et je cherchais à la séduire par tous les moyens. Puis quand j'ai commencé à m'intéresser à la Communauté, j'ai vite tourné la page, elle n'était pas si intéressante que ça en fin de compte. En fait, $\mathrm{j}$ 'ai vite compris mon problème, je faisais

14. Dans son récent ouvrage, Eva Illouz traduit l'expression du self-help par «l'aide à soi-même» dans la mesure où ces techniques «ont pour objet d'améliorer la performativité de l'individu en matière d'intersubjectivité et ne relève [sic] que peu des pratiques new age souvent désignées par le vocable "développement personnel" trop vague» (Illouz, 2012: 11). 
une One-itis, comme chacun d'entre nous. Une fois que j'ai compris les mécanismes de cette dépendance psychologique qui n'a absolument rien en commun avec l'amour, j'ai pu m'en libérer. Ça a pris un peu de temps, mais avec l'aide de certains... Tom surtout. Il avait eu le même problème que moi et il a beaucoup parlé des problèmes d'addiction. Avec son aide, il m'a remis les idées claires et j'ai pu avancer pour arriver là où j'en suis aujourd'hui!

- Et tu en es où?

- Je pense que je suis arrivé à un certain niveau où j'ai appris à prendre du recul et où je contrôle mieux mes émotions. [Sam, 19 ans, étudiant en arts appliqués, région parisienne].

L'entrée dans la Communauté de la séduction est ainsi évoquée sur le mode de la rémission de ce qui passe pour une addiction. Sam fait ainsi une distinction entre ce qu'il considère comme de l' "amour» et le One-itis qui ne relèverait pas du registre des affections mais de celui des addictions, plus communément associées à la passion. L'attachement qui résulte de l'obsession amoureuse n'est donc pas évalué comme un sentiment authentique. Il serait un leurre, puisqu'au bout du compte, l'objet de cet attachement se révèle être «inintéressant». C'est en cela qu'il passe pour un mécanisme psychique défaillant puisqu'il fait croire au sujet qu'il «aime» tandis qu'il serait affecté d'un trouble dont l'effet est d'altérer la réalité émotionnelle. Par ailleurs, l'évocation dans le témoignage de Sam d'un autre membre de la Communauté de la séduction, Tom, est ici significative. D'une part, l'allusion à un pair illustre à quel point le One-itis est l'affaire du groupe et se résout en son sein. La rémission du One-itis passe ainsi par un épisode de solidarité masculine qui s'organise autour du partage de l'expérience spécifique de l'«addiction amoureuse» et des solutions pour «s'en défaire». D'autre part, la référence à Tom s'explique dans ce contexte par le fait que ce dernier s'est érigé en spécialiste des dépendances depuis la parution d'un article intitulé «Témoignage: accro à la pornographie» dont il est l'auteur et qu'il consacre plus particulièrement à la «cyber-porno-dépendance». Publié sur Frenchtouchseduction. com depuis 2007 et régulièrement mis à jour (la dernière remonte au 9 février 2012), l'article est particulièrement lu par les internautes et fréquemment commenté par les apprentis séducteurs qui l'enrichissent de leur propre témoignage. En voici un extrait:

Salut, je m'appelle [Tom], j'ai 29 ans et j'ai longtemps été accro à la pornographie. Le porno, on en rigole, à 15 ans, on se tape des délires dessus avec ses potes, on se dit que ce n'est pas grave parce que de toute façon tout le monde en regarde. Oui, mais voilà, moi ça a été plus loin, beaucoup trop loin à vrai dire $[. .$.$] .$

[Tout] s'est accéléré avec l'arrivée d'Internet et du haut débit. Là, c'était le nirvana du porn qui s'offrait à l'ado déjà accro que j'étais. Le nirvana, et aussi, un désastreux moyen de claquer du pognon en ligne. Certains de mes amis passaient des sommes assez considérables dans l'achat d'accès mensuels pour télécharger des vidéos sur les sites [...].

J'étais timide et la pornographie n'aidait pas à me désinhiber. En réalité, l'enfermement dans un cercle vicieux est assez insidieux. Le processus est souvent le même: tension soudaine, recherche de vidéo (on cherche toujours LA scène, une actrice bien précise, un 
fantasme, une mise en situation...), masturbation, éjaculation. Une fois la jouissance et les endorphines passées, je revenais à une triste vision de la réalité. J'étais seul, comme une merde, je badais [déprimais] et j'avais une estime de moi des plus basses. Mais je recommençais parfois jusqu'à 3 ou 4 fois dans la journée.

Le jour où j'ai pris conscience de cela, il m'a fallu du temps et des mesures draconiennes. Arrêter la masturbation quotidienne, arrêter le porno, installer un filtre parental sur mon pc et apprendre à écouter ma copine. Je me suis mis à compter chaque jour gagné comme un peu plus de terrain grappillé là où j'avais l'impression d'avoir perdu des journées, des semaines voire des mois entiers de ma vie. J'ai jeté toutes les vidéos pornos de mon disque dur (pour certaines, ça a été dur, vous n’imaginez pas à quel point), évacué mes magazines, mes DVD et j'ai eu enfin envie d'accéder à une sexualité où je n'aurais pas besoin de ça, où je pourrais aussi faire preuve de tendresse, d'affection, de compréhension [...].

Voilà, $j$ 'espère que mon témoignage vous aura appris quelque chose, ou fait réfléchir, ou donné envie de réagir. Je ne suis pas un intégriste en croisade contre le porno. Je voulais juste témoigner sur comment le porno m'a pollué mes relations pendant toute une période de ma vie et comme il m’a épuisé. Par moment j'en ressens encore les effets [...]. (Frenchtouchseduction.com, page consultée le 13 août 2011)

Dans ce récit conçu comme exemplaire d'un processus de rémission, Tom retrace son parcours de ce qu'il autodiagnostique comme un parcours type de «dépendant». Il insiste sur les effets de son trouble dans sa vie personnelle (durant l'enquête, il parlera également des effets sur sa vie professionnelle. Lors d'un entretien, il explique avoir été licencié après s'être fait surprendre durant ses heures de travail en train de télécharger un film pornographique). Il évoque non seulement une déperdition importante en argent et temporelle mais également «énergétique» puisqu’il se dit «épuisé» par les effets de sa dépendance. Par ailleurs, sa dépendance l'aurait «détourné» d'une sexualité «normale», soit relationnelle. Pour s'en remettre, il dit avoir pris des mesures draconiennes qui relèvent des techniques du sevrage par la privation des moyens d'accès à l'objet de la dépendance: installation de filtres parentaux, suppression des sources de visionnage, etc. Si ces mesures curatives passent ici pour efficaces, d'autres méthodes - préventives — sont toutefois privilégiées au sein de la Communauté de la séduction.

Celles-ci relèvent d'un apprentissage plus global du contrôle de soi par le biais de diverses techniques thérapeutiques et sportives qui alimentent le marché du développement personnel. Les récits des interviewés font ainsi état de tout un faisceau de pratiques visant l'amélioration de la gestion de soi par le travail du corps et des émotions. Le régime alimentaire, la pratique sportive intensive, le théâtre ou les cours d'expression corporelle sont ainsi couramment évoqués par les interviewés. Pour certains, la méditation est un moyen privilégié pour parvenir à se maîtriser. C'est le cas d'Oryx qui rend compte de cette pratique au cours d'un entretien:

- Je m'intéresse beaucoup à la pensée extrême-orientale et je pratique les arts martiaux depuis quatre ans, en club. C'est dans ce contexte que je me suis intéressé aux techniques 
de méditation. Disons que j'ai beaucoup lu aussi sur cette question. Pour moi, c'était surtout l'idée de se recentrer sur ses sensations, son corps et sa respiration. Après, chacun sa méthode je dirais. Le but c'est que tu arrives à un état de concentration extrême et ça implique que tu trouves ta propre technique quelque part.

- Mais toi, concrètement, tu fais comment?

- Alors, comment je fais? C'est une sorte de routine maintenant. Je m'assois d'abord par terre, mais tu sais, je ne fais pas grand-chose, enfin, ce n'est pas la question! Je me concentre surtout, je me recentre sur moi. Tout est dans la respiration, il faut arriver à ne plus penser à rien en fait. Pour moi, le plus important c'est l'état de concentration dans lequel tu arrives à rentrer. Oui, je dirais que c'est le principal, parce que c'est un état que tu conserves après.

- C'est-à-dire?

- Je veux dire que c'est une maîtrise que tu as sur toi en général (Oryx, 36 ans, psychoclinicien, Nice).

Plutôt que de définir une conduite spécifique propre à la pratique de la méditation, Oryx décrit un état subjectif qui relève de la sensation intériorisée du contrôle de soi, objectif, de l'exercice. Pour se «guérir» d'une obsession amoureuse, certains mettent en place des méthodes similaires, mêlant des techniques de la concentration à celles de la privation (suppression du contact téléphonique ou de l'affiliation sur les réseaux sociaux tels que Facebook à la personne objet du One-itis). C'est le cas d'un internaute qui, après avoir fait le récit d'une rupture amoureuse de laquelle il ne se remettrait pas, répond aux messages de soutien et de conseils qu'il a reçus des visiteurs du même forum:

Bon bah merci les gars, c'est sympa de voir que d'autres sont passés par là et de voir qu'on est pas tout seul. J'essaie de m'en sortir! J'ai déjà supprimé mon One-itis de mon répertoire et aussi de mon compte Facebook. Après c'est clair! C'est que le début... Merci [Golden Boy] pour ton conseil $(;)$. Pratiquer la méditation ça me ferait du bien je pense [...]. Enfin, faut que je reprenne le contrôle en tout cas! [Spikeseduction.com, page consultée le 16 mars 2009]

Ce message de forum illustre bien comment la rémission d'une One-itis est ici associée à la reprise d'un contrôle sur soi. Cet apprentissage de la gestion de ses émotions passe par différentes pratiques et exercices suivant les conseils livrés sur Internet par d'autres membres qui proposent parfois des listes de lecture d'ouvrages de pop psychologie tels que le livre devenu un classique du genre de Napoleon Hill Réfléchissez et devenez riche (2010 [1937]), passé de «main en main » durant l'enquête par observation participante.

La question de la défection de la dépendance sexuelle et amoureuse telle qu'elle se pose dans la suite de ces récits doit être saisie dans la perspective plus globale de l'émergence puis de la diffusion de la catégorie de la dépendance dans les discours profanes et médicaux. Une partie de cette généalogie consacrée plus particulièrement aux discours sur la "porno-dépendance» est l'objet d'un article du sociologue Florian Voros (2009). Ce dernier dégage plusieurs phases d'un processus qu'il qualifie 
d'«invention» de la dépendance à la pornographie. Nous en distinguerons trois phases particulièrement éclairantes pour notre propos.

Reprenant l'article, référence en la matière, de Levine et Troiden (1988), Florian Voros identifie une première phase autour des années 1970 dans les pratiques d'entraide des Alcooliques Anonymes de Boston qui transposent les moyens de sortie de la dépendance à l'alcool à ceux du sexe et de l'amour. La seconde phase se situe dans les années 1980 lors desquelles la dépendance à la pornographie est associée à un trouble obsessionnel compulsif (TOC) et devient un «trouble du contrôle sexuel» (Voros, 2009: 272). Enfin, à partir des années 1990, émerge la catégorie de la «cyber porno-dépendance» (Cooper, 2000; Griffiths, 2001) pensée comme un type de "polyadépendance» aux nouvelles technologies et à la pornographie. «Le "sexe virtuel” est alors conçu comme une drogue, qui, en tant que telle, constitue toujours une échappatoire illusoire à la réalité» (Voros, 2009: 272). Le « dépendant» est alors appréhendé comme un malade qui serait victime de ses propres dépendances.

Cette généalogie, ici schématisée, permet toutefois de mettre en lumière l'émergence d'un sujet type de la dépendance à la pornographie comme un «homme hétérosexuel psychologiquement déstructuré par une forte consommation de représentations explicites de la sexualité féminine» (Voros, 2009: 272). La dépendance à la pornographie et, parallèlement dans le cas étudié ici, à l'amour, est conçue comme une pathologie hétérosexuelle et un trouble du genre masculin. Dans la mesure où les discours sur la dépendance visent quasi exclusivement les hommes, se dessine ainsi en négatif l'injonction à la maîtrise des affections, des désirs et des consommations comme dispositions masculines «normales». De la sorte, si la dépendance à l'amour et à la sexualité est un trouble spécifique de la gestion des émotions, le dépendant souffre principalement d'un déficit de masculinité.

Tom et Sam expliquent ainsi la souffrance qui résulte de leur dépendance par leur incompétence initiale dans la gestion de leur capital émotionnel et sexuel, incompétence à laquelle ils travaillent par le biais de thérapies diverses comme il a été explicité précédemment. Dans l'extrait cité plus haut, Sam raconte comment sa rencontre avec la Communauté est concomitante d'un processus décrit comme une libération vis-àvis d'une dépendance émotionnelle. Il fait donc un lien entre l'apprentissage de la séduction — comme méthode de rémission — et la gestion des émotions amoureuses: "Je pense que je suis arrivé à un certain niveau où j'ai appris à prendre du recul et où je contrôle mieux mes émotions» (Sam, entretien cité). Plus tard dans l'entretien, ce dernier fait de ces compétences acquises la condition sine qua non du «devenir masculin». À la question posée en entretien sur ce qu'il entend par «son niveau» en séduction, il apporte cette réponse laconique: «La Communauté de la séduction m'a permis de devenir un homme, et être un homme c'est savoir se contrôler» (ibid.).

Les techniques du développement personnel promues au sein de la Communauté de la séduction auraient donc pour effet de rétablir un état émotionnel masculin «normal» et fonctionnent dès lors comme un processus émancipateur des formes de la dépendance en reproduisant la trame d'une socialisation genrée fondée sur l'intério- 
risation du contrôle de ses émotions, de sa sexualité ou encore de ses pratiques de consommation. Outre la normalisation des masculinités, l'intériorisation des catégories du contrôle produit un second effet: elle participe à leur hiérarchisation. En devenant les gestionnaires de leurs émotions, les jeunes hommes sur lesquels a porté l'enquête se positionnent dans l'échelle des masculinités (Connell, 1995). Ils acquièrent ainsi des compétences spécifiques, reconnues au sein de la Communauté de la séduction par l'obtention d'un statut: celui d'initié, distinct de celui des débutants, lesquels sont sujets au One-itis. Les «initiés» obtiennent ainsi une légitimité qui se traduit par un certain nombre d'avantages, tel celui d'enseigner aux nouveaux venus les savoirs qu'ils tirent de leur propre expérience. Il s'agit là d'une forme de reconnaissance sociale forte d'une masculinité «accomplie», leur permettant d'endosser une position d'autorité vis-à-vis des masculinités «en devenir».

\section{CONCLUSION}

Dans son dernier ouvrage, Eva Illouz propose une approche sociologique originale de la souffrance amoureuse (Illouz, 2012). En rupture avec le régime explicatif fondé sur une compréhension essentiellement psychologisante des affections, elle élabore une sociologie de la souffrance amoureuse qu'elle conçoit comme un espace privilégié pour penser la modernité. Pour l'auteure, la raison du malaise amoureux n'est pas à chercher au fin fond des histoires personnelles justifiant des choix et des comportements qui passent pour inappropriés et que le marché du développement personnel se charge de "réparer», mais trouve son explication au cœur même des institutions sociales. Projetant ainsi de «traiter de l'amour comme Marx traita des marchandises, [Eva Illouz entend montrer] que l'amour est produit par des rapports sociaux concrets; que l'amour circule sur un marché d'acteurs en situation de concurrence, et inégaux; et [soutient] que certaines personnes disposent d'une plus grande capacité à définir les conditions dans lesquelles elles sont aimées que d'autres» (Illouz, 2012: 18, nous soulignons). À ces dernières assertions auxquelles nous souscrivons pleinement, cet article montre qu'il convient d'en ajouter une dernière. Dans le programme d'une sociologie de la souffrance amoureuse, la compréhension des manières dont certaines personnes disposent d'une plus grande capacité à définir les conditions dans lesquelles elles souhaitent aimer est centrale.

C'est cette partie du processus de la modernité amoureuse que nous avons tenté de saisir ici, en axant l'analyse sur la manière dont des jeunes hommes réfléchissent aux effets individuels et collectifs de l'amour porté aux femmes sur le masculin et la masculinité. Tandis qu'Eva Illouz s'intéresse essentiellement aux raisons sociologiques du chagrin amoureux qu'éprouvent les femmes, dans cet article, nous avons proposé d'envisager ce qu'il se passe du côté des hommes hétérosexuels. Pour des raisons structurelles qui ont trait aux rapports sociaux de $\operatorname{sex}^{15}$, il s'agissait de penser la souffrance

15. Pour une synthèse de la conceptualisation sociologique de la notion de «rapports sociaux de sexe», voir Devreux et Daune-Richard (1992). 
amoureuse comme non symétrique et ne produisant pas les mêmes effets sur les trajectoires masculines que sur les destins féminins.

En s'intéressant plus particulièrement aux discours spécifiquement masculins sur la douleur amoureuse, cet article avait pour objectif de saisir les logiques de genre à l'œuvre dans leur formulation ainsi que leurs effets sur la production des masculinités. Il montre, d'une part, comment l'expérience de la souffrance amoureuse est évaluée par les enquêtés comme historiquement située dans un contexte «post-égalitaire». Les hommes interrogés distinguent ainsi une première raison sociologique au mal-être amoureux masculin causé par une égalité entre les sexes qui serait entérinée et déjà «dépassée». Ces discours que nous qualifions ici de masculinistes envisagent les émotions amoureuses comme le produit d'un rapport hétérosexuel nécessairement traversé par des enjeux de pouvoir. Le rapport amoureux est donc ici perçu comme l'arène de la lutte des sexes et l'espace de la "reconquête» masculine au-delà de la relation hétérosexuelle, dans la scène sociale globale. Nous avons ainsi montré comment l'amour hétérosexuel fait l'objet d'une forte dépréciation au bénéfice de l'amitié entre hommes visant la consolidation de la solidarité masculine.

Dans l'étude du concept du One-itis, nous avons ensuite envisagé comment, à son paroxysme, la souffrance amoureuse est évaluée par les enquêtés comme une maladie, une forme spécifiquement masculine de dépendance de type émotionnel. Comme nous l'avons analysée, la dépendance est d'abord conçue comme un trouble de l'exercice du contrôle sur soi. À partir des données recueillies lors de l'enquête, nous avons ainsi montré comment l'obsession amoureuse «trahit» un manque de masculinité, que la Communauté de la séduction propose de combler par le biais des techniques de développement personnel. Pour s'en remettre et s'en démettre totalement, l'apprenti séducteur doit «devenir un homme» en incorporant certaines de ses propriétés spécifiques: il doit faire preuve d'autorité dans la relation, être expérimenté en matière de relation hétérosexuelle et maîtriser sa sexualité (Gourarier, 2012: 373-413). Plus globalement, il doit apprendre à gouverner ses émotions.

La guérison du One-itis correspond ainsi à un apprentissage de la mise à distance. Mise à distance des rapports amoureux avec les femmes d'abord, par la dépréciation des émotions qu'elles suscitent au bénéfice du rapport entre hommes. Mise à distance de ses propres émotions ensuite, par l'expérience de la retenue et la discipline de soi. L'étude du One-itis permet ainsi d'analyser conjointement deux modalités du travail de mise à distance des émotions et leurs effets. Pensé depuis la perspective foucaldienne de la subjectivation des catégories du pouvoir (Foucault, 1984; 2001), l'exemple du One-itis contribue à la compréhension empirique de la manière dont s'articulent maîtrise de soi et exercice du contrôle, pouvoir sur soi et rapports de pouvoir.

\section{RÉSUMÉ}

Le One-itis est un jeu de mots issu de l'argot américain qui établit une analogie entre les sentiments amoureux et la maladie, la terminologie des pathologies infectieuses se terminant souvent par le suffixe itis. L'usage de cette expression, synonyme d'«obsession amoureuse» s'inscrit dans un contexte spécifique puisque son invention est le fait de jeunes hommes 
(âgés en moyenne d'une vingtaine d'années) qui se réunissent autour de l'apprentissage de la séduction des femmes. La «Communauté de la séduction», ainsi que la nomment ceux qui s'y agrègent, est apparue à la fin des années 1990 en Californie dans la mouvance des techniques émergentes du développement personnel et du coaching appliquées à la séduction masculine. Fondée sur l'examen empirique des pratiques et des discours amoureux formulés par les jeunes hommes rencontrés durant l'enquête, cette contribution entend penser les modalités de l'articulation entre l'expérience sentimentale douloureuse, la normalisation et la hiérarchisation des masculinités.

Mots clés: amour, capital émotionnel, développement personnel, masculinités, sociabilités et socialisation masculine

\section{ABSTRACT}

The One-itis is a play on words derived from American slang that draws an analogy between the love-related feelings and sickness, the terminology of infectious patologies often ending with the suffix «itis». The use of this expression, synonymous with «obsessive love» is part of a specific context since it has been coined by a group of young men (aged 18 to 35 years) who gather in order to learn to seduce women. This circle, the «Community of seduction», appeared in the late 1990s in California in the wake of emerging self-help and coaching techniques geared towards male seduction. Drawing on empirical observation of the practices and discourse of love of the young men encountered during this investigation, this contribution focuses on the interplay between painful emotional experiences and the standardization and hierarchy of masculinities.

Key words: love, emotional capital, self-help, male sociability and socialisation

\section{RESUMEN}

One-itis es un juego de palabras proveniente del argot estadounidense que establece una analogía entre los sentimientos amorosos y la enfermedad, utilizando la terminología de las enfermedades infeccionas que, con frecuencia, terminan en el sufijo "itis". La utilización de esta expresión, sinónimo de "obsesión amorosa", se inscribe en un contexto específico, cuya creación proviene de la reunión de hombres jóvenes (de una edad promedio de 20 años) alrededor del aprendizaje de la seducción de las mujeres. La "Comunidad de la seducción", tal como es nombrada por quienes se suman a ella, apareció a finales de los años 1990 en California, con el movimiento de las técnicas del desarrollo personal y del coaching, aplicadas a la seducción masculina. Concebida a partir del análisis empírico de las prácticas y del discurso amoroso de jóvenes entrevistados, aquí se realiza una reflexión acerca de las modalidades de la articulación entre la experiencia sentimental dolorosa, la normalización y la jerarquización de las masculinidades.

Palabras claves: amor, capital emocional, desarrollo personal, masculinidades, sociabilidades y socialización masculina 


\section{BIBLIOGRAPHIE}

Anderson, P. T. (2000), Magnolia, New Line Cinema.

Bologne, J. C. (2007), Histoire de la conquête amoureuse. De l'Antiquité à nos jours, Paris, Seuil.

Bourdieu, P. (1979), La distinction, Paris, Éditions de Minuit.

Bozon, M. (2001), «Les cadres sociaux de la sexualité», Sociétés contemporaines, vol. 41-42, nº 1, p. 5-9.

Connell, R. (1995), Masculinities, Cambridge, Polity Press.

Cooper, A. (2000), Cybersex: The Dark Side of the Force. A Special Issue of the Journal of Sexual Addiction and Compulsion, New York, Routledge.

Delphy, C. (2010), Un universalisme si particulier. Féminisme et exception française (1980-2010), Paris, Syllepse.

Delumeau, J. (1978), La Peur en Occident (XIVe-XVIII siècles): une cité assiégée, Paris, Fayard.

Devreux, A. M et A. M. Daune-Richard (1992), "Rapports sociaux de sexe et conceptualisation sociologique», Recherches féministes, vol. 5, $\mathrm{n}^{\circ} 2$, p. 7-32.

Duby, G. (1988), Mâle Moyen Âge: de l'amour et autres essais, Paris, Flammarion.

Elias, N. (1973), La Civilisation des mours, Paris, Calmann-Lévy.

Forgeau-Zerbib, F. (2001), «L'égalité des sexes institutionnalisée? Des politiques publiques aux rapports de séduction, un examen du «modèle» norvégien », Thèse de sociologie, Université de Saint-Quentinen-Yvelines.

Foucault, M. (1984), (2001), L’Herméneutique du sujet, Paris, Gallimard.

- (2008), Histoire de la sexualité 3. Le souci de soi, Paris, Gallimard.

Febvre, L. (1943), "La sensibilité dans l'histoire: les courants collectifs de pensée et d'action », in La Sensibilité dans l'homme et dans la nature, Paris, $10^{\text {e }}$ Semaine internationale de synthèse, 7-12 juin 1938, p. 77-106.

Frevert, U. (2009), «Was haben Gefühle in der Geschichte zu suchen?», Geschichte und Gesellschaft, vol. $35, \mathrm{n}^{\circ} 2$, p. $183-208$.

Goethe, J. W. (1999 [1774]), Les souffrances du jeune Werther, Paris, Gallimard.

Gourarier, M. (2012), «Séduire les femmes pour s'apprécier entre hommes. Une ethnographie des sociabilités masculines hétérosexuelles au sein de la Communauté de la séduction en France», Thèse d'anthropologie sociale et ethnologie, Paris, EHESS.

_- (2011), «Négocier le genre. Une ethnologue dans une société d'hommes apprentis séducteurs», Journal des anthropologues, $\mathrm{n}^{\circ} 124-125$, p. 159-178.

- , (2007), «Le speed dating en France. Une sociologie de la rencontre amoureuse hétérosexuelle», mémoire de DEA d'anthropologie sociale, Paris, EHESS.

Gray, J. (1997 [1992]), Les hommes viennent de Mars, les femmes viennent de Vénus, Paris, Éditions du Collectionneur.

Griffiths, M. (2001), «Sex on the Internet: Observations and Implications for Internet Sex Addiction», The Journal of Sex Research, vol. 38, n 4, p. 333-342.

Hill, N. (2010 [1937]), Réfléchissez et devenez riche, Paris, J'ai lu.

Hochschild, A. R. (1983), The Managed Heart: The Commercialization of Human Feeling, Berkeley, The University of California Press.

_ - (2003), «Travail émotionnel, règles de sentiments et structure sociale», Travailler, vol. 9, $\mathrm{n}^{\circ} 1$, p. 19-49.

Illouz, E. (2012), Pourquoi l'amour fait mal. L'expérience amoureuse dans la modernité, Paris, Seuil.

- (2006), Les sentiments du capitalisme, Paris, Seuil.

JonAs, I. (2006), «L'antiféminisme des nouveaux “traités de savoir-vivre à l'usage des femmes” ", Nouvelles questions féministes, vol. $25, \mathrm{n}^{\circ} 2$, p. 82-95.

Kauffman, J.-C. (1999), La femme seule et le prince charmant, Paris, Nathan.

La Fayette, Mme de (2009 [1678]), La Princesse de Clèves, Paris, Flammarion. 
Levine, M. P. and R. R. Troiden (1988), «The Myth of Sexual Compulsivity», The Journal of Sex Research, vol. $25, \mathrm{n}^{\circ} 3$, p. 347-363.

Monneyron, F. (1997), Séduire, l'imaginaire de la séduction de Don Giovanni à Mick Jagger, Paris, PUF.

Rougemont, D. de (1972), L'amour et l'Occident, Paris, Éditions du 10/18.

Singly, F. de (2011), Séparée. Vivre l'expérience de la rupture, Paris, Armand Colin.

Voros, F. (2009), «L'invention de l'addiction à la pornographie», Sexologies, nº 18, p. 270-276.

\section{Sites Internet consultés:}

www.frenchtouchseduction.com

www.naturaltechnics.com

www.spikeseduction.com

www.verselejus.com 